PHYSICAL REVIEW A 101, 020302(R) (2020)

Rapid Communications

\title{
Berry phase estimation in gate-based adiabatic quantum simulation
}

\author{
Bruno Murta, ${ }^{*}$ G. Catarina $\odot$, and J. Fernández-Rossier ${ }^{\dagger}$ \\ QuantaLab, International Iberian Nanotechnology Laboratory (INL), Avenida Mestre José Veiga, 4715-330 Braga, Portugal
}

(Received 26 September 2019; revised manuscript received 16 January 2020; accepted 16 January 2020; published 11 February 2020)

\begin{abstract}
Gate-based quantum computers can in principle simulate the adiabatic dynamics of a large class of Hamiltonians. Here, we consider the cyclic adiabatic evolution of a parameter in the Hamiltonian. We propose a quantum algorithm to estimate the Berry phase and use it to classify the topological order of both single-particle and interacting models, highlighting the differences between the two. This algorithm is extensible to an interacting topological system. Our results evidence the potential of near-term quantum hardware for the topological classification of quantum matter.
\end{abstract}

DOI: 10.1103/PhysRevA.101.020302

Fault-tolerant universal quantum computers are expected to efficiently simulate the unitary evolution of large classes of quantum Hamiltonians [1-3], including those relevant for condensed matter [4], quantum chemistry [5], and subatomic physics [6]. In particular, they will help to address the exponential wall problem [7] faced in the simulation of quantum many-body phenomena.

Algorithms for the preparation of complicated quantum states are required in most digital quantum simulation (DQS) strategies. In some instances, such as hybrid variational methods [8] and phase estimation [9], the preparation of approximate quantum states is a valid approach, as long as the overlap with the target exact state is large enough. However, this overlap is expected to become exponentially small as the number of degrees of freedom increases [10]. A solution to this problem is parametric adiabatic evolution via DQS [11]. Starting from a Hamiltonian for which the ground state can be easily obtained, the extra terms are added slowly, and, by virtue of the adiabatic theorem [12], the quantum state of the system stays in the ground state of the new Hamiltonian.

A central concept in the theory of adiabatic parametric evolution is the Berry phase [13]. As a Hamiltonian is cycled adiabatically around a closed path in a parameter space, the wave function acquires a geometric phase [13] in addition to the dynamical phase. The Berry phase plays a crucial role in several domains of quantum theory [14], including our understanding of the electronic properties of molecules [15], nanomagnets [16,17], solids [18,19], and the topological theory of quantum matter [20,21]. Specifically, the Berry phase can be used as a quantized index for the topological classification of different classes of Hamiltonians, including one-dimensional symmetry-protected topological insulators [22-24], gapped spin liquids [25], and interacting fermion models [26].

As one of the main platforms for quantum simulation, superconducting qubits have been used to explore topological

\footnotetext{
*bruno.murta@inl.int

${ }^{\dagger}$ Also at Departamento de Física Aplicada, Universidad de Alicante, 03690 San Vicente del Raspeig, Alicante, Spain.
}

states. Quantum algorithms to measure single-particle topological invariants, one based on quantum walks [27] and another for finite temperatures [28], have been recently proposed. A more general method was used to probe topological transitions in both single-qubit [29] and coupled two-qubit [30] systems. This involved the measurement of deflections from the adiabatic path to obtain the local Berry curvature [31], which was then integrated to obtain the Berry phase.

Here, we propose a quantum algorithm that yields the Berry phase without requiring the explicit integration of the Berry curvature. Our algorithm combines phase estimation and gate-based simulation of adiabatic quantum evolution to obtain the Berry phase, as opposed to the so-called adiabatic quantum computing [32]. This algorithm can be applied to a wide class of Hamiltonians in a parameter space. In particular, we show how it can be used for the topological classification of model Hamiltonians with gapped ground states, working out the cases of both the paradigmatic Su-Schrieffer-Heeger (SSH) Hamiltonian [33] for independent fermions and the dimerized Heisenberg $S=1 / 2$ spin chain [34].

The formal statement of the problem addressed here is the following. Given a family of Hamiltonians $\mathcal{H}(\rho)$ obtained from variations of a parameter $\rho$, we focus on the case where, for every $\rho, \mathcal{H}(\rho)$ has a nondegenerate ground state $\left|\Psi_{G}(\rho)\right\rangle$ with energy $E_{G}(\rho)$. At $t=0, \rho(t=0) \equiv \rho_{0}$, and the system is prepared in its ground state $\left|\Psi_{G}\left(\rho_{0}\right)\right\rangle$. The system evolves in time as $\rho$ changes slowly enough to ensure that it remains in the ground state $\left|\Psi_{G}(\rho)\right\rangle$ per the adiabatic theorem [12]. After a time $T, \rho=\rho_{T}$ and $\mathcal{H}\left(\rho_{T}\right)=\mathcal{H}\left(\rho_{0}\right)$. Without loss of generality $\rho$ can be considered to be an angle that varies between 0 and $2 \pi$ and $\mathcal{H}$ to depend on $\rho$ via periodic functions. The parametric evolution can thus be visualized as a loop in the unit circle generated by $\rho \in[0,2 \pi)$.

The quantum state at $t=T$ adopts the form

$$
\left|\Psi_{G}(2 \pi)\right\rangle=e^{-i \theta_{D}} e^{i \theta_{B}}\left|\Psi_{G}(0)\right\rangle,
$$

where $\theta_{D}=\frac{1}{\hbar} \int_{0}^{T} E_{G}[\rho(t)] d t$ is the dynamical phase and

$$
\theta_{B}=-i \int_{0}^{2 \pi}\left\langle\Psi_{G}(\rho) \mid \frac{\partial \Psi \Psi_{G}(\rho)}{\partial \rho}\right\rangle d \rho
$$


(a) $|0\rangle$

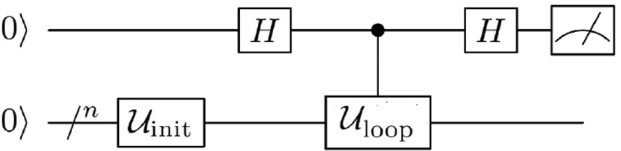

(b) $|0\rangle$

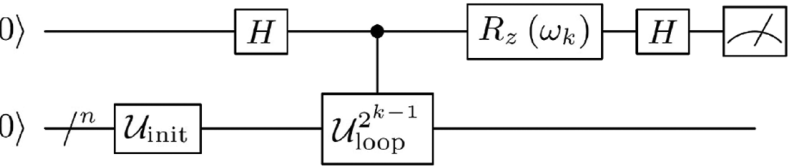

FIG. 1. Quantum circuits to measure Berry phase. $\mathcal{U}_{\text {init }}$ represents the subcircuit that initializes the $n$-qubit register in an eigenstate of $\mathcal{H}$. $\mathcal{U}_{\text {loop }}$ implements the quantum simulation of the adiabatic loop. $H$ are Hadamard gates. (a) Hadamard-test scheme [35]. (b) Iterative phase estimation (IPE) scheme [36]. The quantum circuit shows a $k$ th iteration. The $R_{z}\left(\omega_{k}\right)$ gate, where $\omega_{k}=-2 \pi 0.0 \cdots \gamma_{k+1} \cdots \gamma_{R}$ and $R$ is the total number of iterations, serves to remove the contribution to the phase from the previously measured bits.

is the Berry phase. Our goal is to carry out a gate-based quantum simulation of the adiabatic loop to determine $\theta_{B}$. This is accomplished by a combination of quantum phase estimation [2] and gate-based quantum simulation of the adiabatic evolution. The proposed quantum circuits are shown in Fig. 1.

We first discuss the circuit shown in Fig. 1(a), which represents the standard interferometric phase estimation circuit [35]. An ancilla qubit reads out the Berry phase, and an $n$-qubit register stores the quantum state that undergoes the evolution. The initialization subroutine, denoted by $\mathcal{U}_{\text {init }}$, accomplishes $\mathcal{U}_{\text {init }}|0\rangle_{n}=\left|\psi_{G}\right\rangle$. For the examples considered in this Rapid Communication, the exact initial state can be found via exact diagonalization. In general, however, this $\mathcal{U}_{\text {init }}$ subroutine may correspond to another quantum algorithm such as quantum phase estimation [2] or the variational quantum eigensolver (VQE) [37].

The crux of the matter lies on the second stage, which carries out the controlled adiabatic evolution $\mathcal{U}_{\text {loop }}\left|\psi_{G}\right\rangle=$ $e^{i \phi}\left|\psi_{G}\right\rangle$, where $\phi$ depends both on the dynamical and the Berry phases. Performing an adiabatic evolution for the state initialization prior to the phase estimation scheme has been previously explored, but instead we introduce the adiabatic evolution within the phase estimation itself. The combination of a Hadamard gate on the ancilla and a controlled operation kick the $\phi$ phase onto the top register. As a result, in the last stage of the process, the probability of the ancilla being measured as 0 is $P_{0}=\cos ^{2}\left(\frac{\phi}{2}\right)$ (see Supplemental Material [38] and Ref. [35]).

In this Rapid Communication we also use the so-called iterative phase estimation algorithm (IPEA) [36], the output of which is the phase itself expressed as an $R$-bit binary fraction of the form $\frac{\phi}{2 \pi}=\sum_{k=1, R} \frac{\phi_{k}}{2^{k}}$, where $R$ is the number of iterations. The binary digits $\phi_{k}$ are obtained by repeatedly applying the circuit at the bottom of Fig. 1. $R_{z}\left(\omega_{k}\right)$ uses the results of the previous steps to gauge away the corresponding phase in the ancilla qubit, thus ensuring that at the $k$ th iteration the circuit yields the digit $\phi_{R-k}$.
A crucial element of the generality of our algorithm lies in the structure of $\mathcal{U}_{\text {loop. }}$. Specifically, we can take

$$
\mathcal{U}_{\text {loop }}=U_{\circlearrowleft}(0, T / 2) U_{\circlearrowleft}(T / 2,0),
$$

where the first (second) argument stands for forward evolution in time from $t=0$ to $t=T / 2$ (backward evolution in time from $t=T / 2$ to $t=0$ ) and the subindex $\circlearrowleft$ denotes a counterclockwise cycle in $\rho$ space. In words, in the first half of the cycle $\rho$ changes counterclockwise from 0 to $\pi$, but time evolves forward, and in the second half $\rho$ changes counterclockwise from $\pi$ to $2 \pi$ and time goes backward.

Provided that

$$
\int_{0}^{\pi} E_{G}(\rho) d \rho=\int_{\pi}^{2 \pi} E_{G}(\rho) d \rho
$$

the dynamical phase is canceled. Equation (4) is satisfied by definition in time-reversal invariant Hamiltonians.

The implementation of the adiabatic evolution quantum subroutine $\mathcal{U}_{\text {loop }}$ in gate-based quantum computers, such as the IBM Q Experience devices, is accomplished by breaking down the evolution in $N$ steps of duration $\delta t$ during which $\rho$ stays constant,

$$
\mathcal{U}_{\text {loop }}=\prod_{j=1}^{N} \delta \mathcal{U}\left(\rho_{j}\right) .
$$

Here, $\delta \mathcal{U}\left(\rho_{j}\right)=\exp \left[-i \mathcal{H}\left(\rho_{j}\right) \delta t / \hbar\right]$ stands for the unitary propagator element due to the Hamiltonian $\mathcal{H}\left(\rho_{j}\right)$, keeping $\rho$ constant. The choice of both $T$ and $N$ is determined by two competing factors. On the one hand, the adiabatic condition requires that $\frac{\delta \rho}{\delta t}=\frac{2 \pi}{T}$ is small, which imposes large enough $N$ (cf. $\delta \rho=\frac{2 \pi}{N}$ ) and $T$. On the other hand, the number of gates in the quantum simulation algorithm increases with both $N$ and $T$, which is an issue given the limitations of current quantum hardware.

We now substantiate our proposal by describing the quantum circuit that implements $\mathcal{U}_{\text {loop }}$ in the context of the topological classification of quantum phases of two different model Hamiltonians. We first consider the SSH model, which describes a one-dimensional tight-binding model for spinless fermions with one orbital per site, intracell hopping $v$, and intercell hopping $w$ [Fig. 2(a)]. Using Bloch's theorem, the Hamiltonian can be block-diagonalized in terms of $2 \times 2$ matrices.

$$
\mathcal{H}_{\mathrm{SSH}}(k)=(v+w \cos k) \sigma_{x}+(w \sin k) \sigma_{y} \equiv \vec{h}(k) \cdot \vec{\sigma},
$$

where $k$ is the wave number. For $v \neq w$ this model describes an insulator, with two energy bands separated by a gap of size $2|v-w|$ at $k= \pm \pi$. The different topological nature of these two phases is characterized by a specific case of Berry phase, known as the Zak phase [39], which is obtained when the ground state of $\mathcal{H}_{\mathrm{SSH}}(k)$ is looped in $k$ space across the first Brillouin zone. The Berry phase can be used as a topological index,

$$
\theta_{B}=-i \int_{0}^{2 \pi}\left\langle\Psi_{G}(k) \mid \frac{\partial \Psi \Psi_{G}(k)}{\partial k}\right\rangle d k= \begin{cases}\pi, & v<w, \\ 0, & v>w .\end{cases}
$$

The number of in-gap edge modes is given by $2 \theta_{B} / \pi$, so that only the $v<w$ phase has robust in-gap edge states and is 


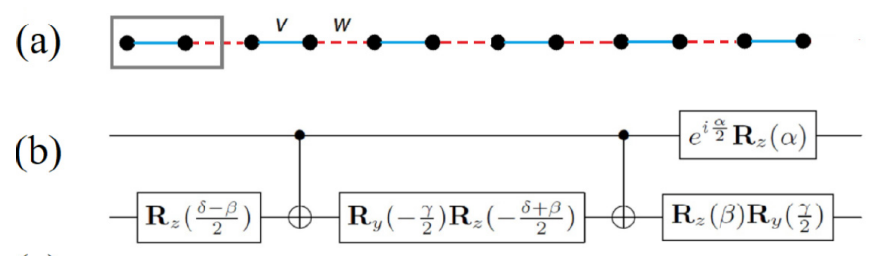

(c)

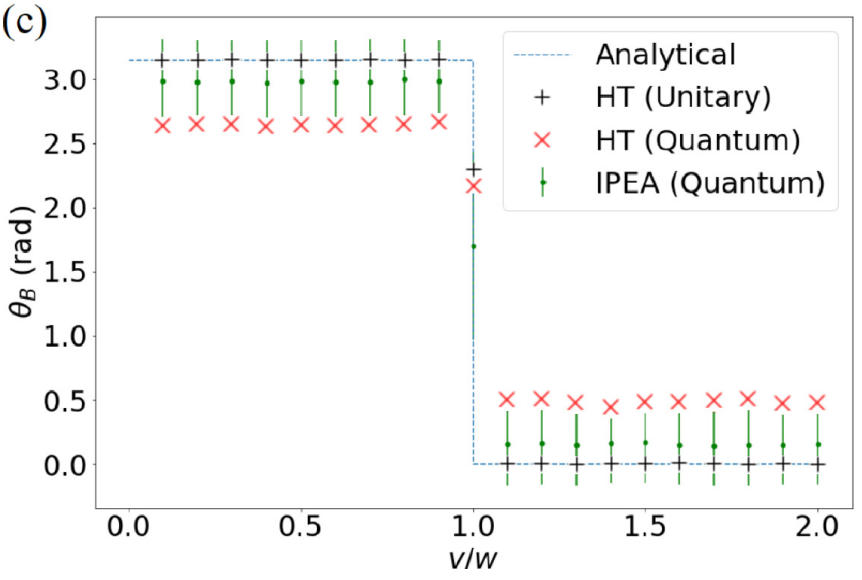

FIG. 2. (a) SSH tight-binding chain with intra- and intercell hopping parameters $v$ and $w$. The box delimits a unit cell. (b) Quantum circuit implementing a controlled- $\delta \mathcal{U}$ gate defined in Eq. (5). $\alpha, \beta$, $\gamma$, and $\delta$ are related to the parameter $\rho$ and the Hamiltonian $\mathcal{H}_{\mathrm{SSH}}$ as described in the Supplemental Material [38]. (c) Berry phase, as obtained from Eq. (7) analytically (dashed line), in silico unitary simulation (black pluses), and experimental quantum simulation (red crosses) of the Hadamard-test (HT) circuit [Fig. 1(a)], and quantum simulation of the IPEA circuit [Fig. 1(b)] for $R=4$ iterations and $N=4$ time steps (green dots with respective error bars). Quantum simulations were carried out in the ibmq_16_melbourne device from the IBM Q Experience. Further details about the quantum simulation can be found in the Supplemental Material.

said to be topological. This is a manifestation of the bulkboundary correspondence $[20,21]$. Hence, at $v=w$ there is a topological phase transition as a topological invariant changes value.

Taking the SSH model to reciprocal space permits several simplifications. First, the wave function can be encoded in a single qubit. Second, the controlled unitary operations can be implemented by taking advantage of closed-form analytical expressions for the unitary evolution operator (see Supplemental Material [38]). Also note that $E_{G}(k)=E_{G}(-k)$, so Eq. (4) is satisfied and the dynamical phase is canceled via the time-reversal trick. The Berry phase for the SSH model as a function of $v / w$ is shown in Fig. 2(c) as obtained in four different ways: analytically [Eq. (7)], via an in silico simulation of the Hadamard-test circuit shown in Fig. 1(a), and via the implementation of both circuits in Fig. 1 on the ibmq_16_melbourne device [40]. The controlled- $\delta \mathcal{U}$ gate was implemented using the circuit of Fig. 2(b) in both the unitary simulation and the actual quantum computations.

The results for the Hadamard-test circuit in quantum hardware (red markers) are close but not quite within the (shot-noise) error range from the analytical values for $N=4$ time steps. This is due to the limitations of current quantum hardware. We have verified that IPEA [Fig. 1(b)] gives results closer to the theory with $R=4$ iterations [see the green markers in Fig. 2(c)]. Naturally, for $R$ iterations the maximum precision that can be achieved is $2^{-R}$, while in the original method the precision is shot-noise bounded. The choice between the Hadamard-test circuit and IPEA therefore involves a trade-off between accuracy and precision.

The topological classification of noninteracting models can be efficiently done with classical computers. This is, however, not the case for interacting systems for which there are no analytical solutions and whose size is beyond the capacity of conventional computers. We now show that our algorithm can be used in this second class of nontrivial systems. To do so, we implement our proposal to address the topological classification of the ground state of the dimerized Heisenberg spin chain. The Hamiltonian for periodic boundary conditions (PBCs) reads as

$$
\hat{H}_{\mathrm{PBC}}=\sum_{i=0}^{N_{s} / 2-1}\left(\frac{J_{+}}{4} \vec{\sigma}_{2 i+1} \cdot \vec{\sigma}_{2 i+2}+\frac{J_{-}}{4} \vec{\sigma}_{2 i+2} \cdot \vec{\sigma}_{2 i+3}\right),
$$

where $J_{ \pm}=J \pm \delta, J$ is the average spin coupling, $\delta$ is the dimerization parameter, $N_{s}$ is the number of $S=1 / 2$ spins in the chain, $\vec{\sigma}_{i}=\left(\sigma_{i}^{x}, \sigma_{i}^{y}, \sigma_{i}^{z}\right)^{T}$ is the Pauli vector for the $i$ th spin, and $\vec{\sigma}_{N_{s}+1}=\vec{\sigma}_{1}$ due to the periodicity. The Hamiltonian for open boundary conditions (OBCs) reads $\mathcal{H}_{\mathrm{OBC}}=\mathcal{H}_{\mathrm{PBC}}-$ $\frac{J_{-}}{4} \vec{\sigma}_{N} \cdot \vec{\sigma}_{1}$. This Hamiltonian has only been solved analytically for the case $\delta=0[41,42]$, the well-known gapless spin liquid. For $\delta \neq 0$, reliable information is based on density matrix renormalization group [43] and exact diagonalizations [34]. As for the SSH Hamiltonian, the OBC chain has in-gap edge excitations for $\delta<0$, but not for $\delta>0$. This, together with the fact that this model can be obtained from the SSH model when strong Hubbard repulsion is added [44,45], implies the two phases are topologically different.

The topological classification of the model can be done using a method proposed by Hatsugai [25], which consists of introducing a twist phase $\rho$ in a single local bond,

$$
\vec{\sigma}_{j-1} \cdot \vec{\sigma}_{j} \rightarrow \frac{1}{2}\left(e^{-i \rho} \sigma_{j-1}^{+} \sigma_{j}^{-}+e^{i \rho} \sigma_{j-1}^{-} \sigma_{j}^{+}\right)+\sigma_{j-1}^{z} \sigma_{j}^{z} .
$$

The PBC ground state remains nondegenerate and gapped as $\rho$ is ramped between 0 and $2 \pi$ in the ring geometry. The Berry phase $\theta_{B}(j)$ that arises from this $\rho$ loop defines a local topological marker that reveals the dimer structure of the chain: It is $\pi$ at the stronger links and 0 at the weaker ones [Fig. 3(a)]. As $\delta$ changes sign and a strong bond becomes a weak one, the corresponding local Berry phase goes from $\pi$ to 0 , and vice versa. Crucially, if a strong bond is removed from the $\mathrm{PBC}$ ring, the resulting $\mathrm{OBC}$ chain is topologically nontrivial due to the presence of topologically protected edge states. If instead a weak bond is removed, no edge states appear.

To implement the Berry phase estimation algorithm, several technical caveats that were absent in the case of the SSH model have to be dealt with. First, we need as many qubits as sites in the spin chain. Remarkably, the topological transition survives in small systems with as few as four spins, although finite-size effects are present (see Supplemental Material [38]). Second, the ground state $\left|\Psi_{G}(\rho=0)\right\rangle$, which is no longer a product state in the computational basis, must be initialized before the start of the adiabatic loop. This is 


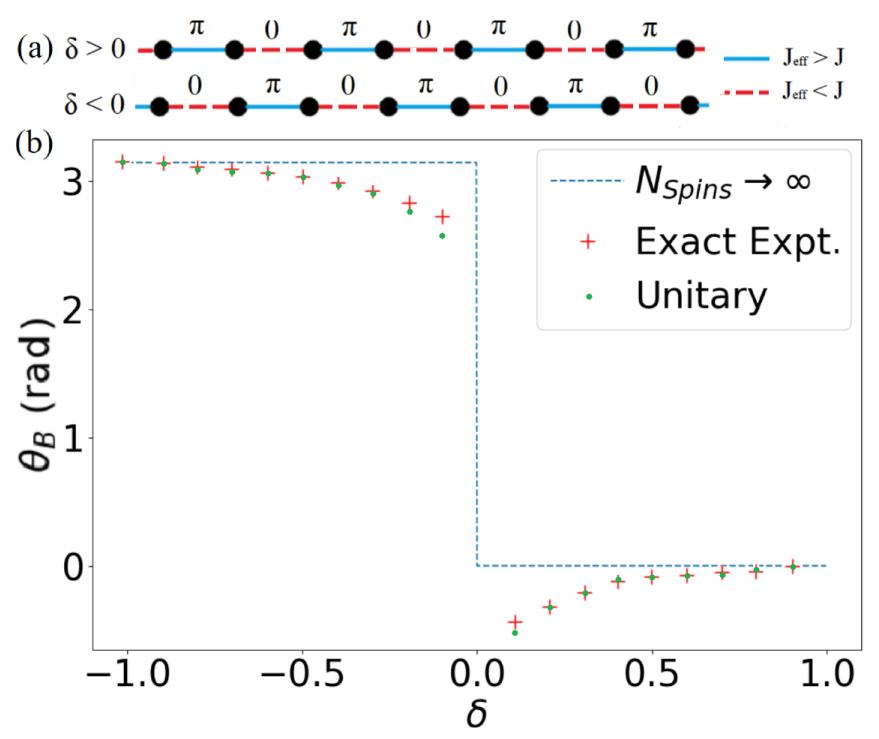

FIG. 3. (a) Local Berry phase $\theta_{B}$ obtained via a Hatsugai twist [25] as a local topological marker that reveals the dimer structure of the Heisenberg ring. Strong links $\left(J_{\text {eff }} \equiv J \pm \delta>J\right)$ have $\theta_{B}=\pi$, while weak ones $\left(J_{\text {eff }}<J\right)$ have $\theta_{B}=0$. (b) Local Berry phase of a link with coupling $J_{\text {eff }}=J-\delta$ for a dimerized Heisenberg ring of four spins with $J=1$ obtained via a noiseless unitary simulation of the Berry phase estimation circuit shown in Fig. 1(a). Quantum algorithm results (green dots) deviate slightly from those obtained via exact exponentiation of the full $16 \times 16$ Hamiltonian (red pluses) due to shot-noise and Trotterization errors. Both simulations reveal a deviation from the expected steplike pattern (blue dashed line) due to finite-size effects (see Supplemental Material [38]).

accomplished in two stages: Obtaining $\left|\Psi_{G}(\rho=0)\right\rangle$ as a linear combination of computational basis states via numerical diagonalization of the model, followed by the preparation of the state using the approach proposed by Shende et al. [46]. The number of gates required for this initialization varies depending on the specific values of $J, \delta$, and $\rho$, taking values between 44 and 79 for a four-spin ring. The number of controlled-NOT (CNOT) gates corresponds to roughly two thirds of the total number of gates. Third, the Hamiltonian is the sum of noncommuting terms, so the implementation of the propagator requires a Trotter-Suzuki expansion [47,48]. Last, the decomposition of the controlled propagator in terms of basis gates cannot be achieved via the $Z-Y-Z$ decomposition as before, since the input register involves more than one qubit. Instead, we make use of a scheme proposed in Ref. [49] (see Supplemental Material [38]). Regarding the cancellation of $\theta_{D}$, it can be checked numerically that the spectrum satisfies Eq. (4) for the Hatsugai twist.

The results of the in silico simulation of the Berry phase estimation algorithm [Hadamard-test circuit shown in Fig. 1(a)] applied to the topological classification of the dimerized Heisenberg ring are shown in Fig. 3(b) (green markers), for a system with $N_{s}=4$ spins and $J=1$, as $\delta$ is ramped. These results differ only slightly from those obtained from the numerical simulation in classical hardware where the propagator of the full Hamiltonian is obtained via exact exponentiation (orange markers). This minor discrepancy is due to shot-noise and Trotterization errors. Both sets of results deviate from the expected steplike pattern (blue dashed line) due to finite-size effects (see Supplemental Material [38]).

Finally, we discuss the perspective for implementation of the topological classification algorithm in quantum hardware. As a preliminary step, two sanity checks on the ground-state initialization for a ring of four spins were carried out. First, its energy was estimated via IPEA [36] with $R=8$ in a noiseless simulation; good agreement with the exact diagonalization results was observed. Second, a parity conservation check [50] was conducted in both a noiseless simulation and the ibmq_16_melbourne device. The results, shown in Fig. 6 of the Supplemental Material [38], show a clear discrepancy between the noiseless simulation and the actual quantum experiment, implying that the $\mathcal{U}_{\text {init }}$ subroutine alone is too deep for current quantum hardware even for just four spins.

In principle, our Berry phase estimation quantum algorithm method can be used to implement the topological classification scheme proposed by Hatsugai [25] in higherspin systems, in higher dimensions, and also for fermions in general [26]. Hence, we hope that the proposed Berry phase estimation algorithm will be used as a tool to explore interacting topological phases when digital quantum computers outperform conventional computers in the simulation of quantum systems. There are, however, a few issues that need to be addressed to extend the applicability of this algorithm, notably canceling the dynamical phase without having to satisfy Eq. (4). Moreover, adiabatic evolution typically leads to deep quantum circuits that defy the coherence properties of current quantum processors. The optimization of the adiabatic evolution [51] and the conversion of a single deep quantum circuit into multiple shallow ones [52] - in line with the recent success of hybrid variational algorithms [37,53]—should be considered.

In summary, we have proposed a quantum algorithm to estimate the Berry phase acquired during the digitized quantum simulation of the ground state of an Hamiltonian as it undergoes an adiabatic loop in a parameter space. Our approach combines the phase estimation algorithm $[35,36]$ with the gate-based quantum simulation of cyclic adiabatic evolutions to estimate the Berry phase. We have discussed the use of this algorithm to classify topological phases of two types of Hamiltonians: The SSH model and the dimerized Heisenberg spin model in one dimension (1D). We have also successfully implemented the algorithm in IBM quantum hardware, evidencing the topological phase transition of the SSH chain. This Rapid Communication illustrates the potential of digital quantum computing to simulate topological quantum many-body systems.

We acknowledge P. Cruz and J. Luis Lado for fruitful discussions. B.M. and J.F.R. acknowledge the FCT Functionalized Graphene for Quantum Technologies project (PTDC/FIS-NAN/4662/2014). G.C. acknowledges support from Fundação para a Ciência e Tecnologia (FCT) Ph.D. Scholarship No. SFRH/BD/138806/2018. All authors acknowledge use of the IBM Q for this work. The views expressed are those of the authors and do not reflect the official policy or position of IBM or the IBM Q team. 
[1] D. S. Abrams and S. Lloyd, Phys. Rev. Lett. 79, 2586 (1997).

[2] D. S. Abrams and S. Lloyd, Phys. Rev. Lett. 83, 5162 (1999).

[3] D. Wecker, M. B. Hastings, N. Wiebe, B. K. Clark, C. Nayak, and M. Troyer, Phys. Rev. A 92, 062318 (2015).

[4] J. Zhang, M.-H. Yung, R. Laflamme, A. Aspuru-Guzik, and J. Baugh, Nat. Commun. 3, 880 (2012).

[5] B. P. Lanyon, J. D. Whitfield, G. G. Gillett, M. E. Goggin, M. P. Almeida, I. Kassal, J. D. Biamonte, M. Mohseni, B. J. Powell, M. Barbieri, A. Aspuru-Guzik, and A. G. White, Nat. Chem. 2, 106 (2010).

[6] E. F. Dumitrescu, A. J. McCaskey, G. Hagen, G. R. Jansen, T. D. Morris, T. Papenbrock, R. C. Pooser, D. J. Dean, and P. Lougovski, Phys. Rev. Lett. 120, 210501 (2018).

[7] W. Kohn, Rev. Mod. Phys. 71, 1253 (1999).

[8] A. Kandala, A. Mezzacapo, K. Temme, M. Takita, M. Brink, J. M. Chow, and J. M. Gambetta, Nature (London) 549, 242 (2017).

[9] A. Aspuru-Guzik, A. D. Dutoi, P. J. Love, and M. Head-Gordon, Science 309, 1704 (2005).

[10] P. W. Anderson, Phys. Rev. Lett. 18, 1049 (1967).

[11] R. Barends, A. Shabani, L. Lamata, J. Kelly, A. Mezzacapo, U. Las Heras, R. Babbush, A. G. Fowler, B. Campbell, Y. Chen et al., Nature (London) 534, 222 (2016).

[12] M. Born and V. Fock, Z. Phys. 51, 165 (1928).

[13] M. V. Berry, Proc. R. Soc. Lond. A 392, 45 (1984).

[14] F. Wilczek and A. Shapere, Geometric Phases in Physics (World Scientific, Singapore, 1989).

[15] R. Resta, J. Phys.: Condens. Matter 12, R107 (2000).

[16] J. von Delft and C. L. Henley, Phys. Rev. Lett. 69, 3236 (1992).

[17] W. Wernsdorfer and R. Sessoli, Science 284, 133 (1999).

[18] D. Xiao, M. C. Chang, and Q. Niu, Rev. Mod. Phys. 82, 1959 (2010).

[19] D. Vanderbilt, Berry Phases in Electronic Structure Theory: Electric Polarization, Orbital Magnetization and Topological Insulators (Cambridge University Press, Cambridge, UK, 2018).

[20] M. Z. Hasan and C. L. Kane, Rev. Mod. Phys. 82, 3045 (2010).

[21] X.-L. Qi and S.-C. Zhang, Rev. Mod. Phys. 83, 1057 (2011).

[22] P. Delplace, D. Ullmo, and G. Montambaux, Phys. Rev. B 84, 195452 (2011).

[23] T. Cao, F. Zhao, and S. G. Louie, Phys. Rev. Lett. 119, 076401 (2017).

[24] C. G. Velasco and B. Paredes, Phys. Rev. Lett. 119, 115301 (2017).

[25] Y. Hatsugai, J. Phys. Soc. Jpn. 75, 123601 (2006).

[26] N. H. Le, A. J. Fisher, N. J. Curson, and E. Ginossar, arXiv:1906.00488.

[27] E. Flurin, V. V. Ramasesh, S. Hacohen-Gourgy, L. S. Martin, N. Y. Yao, and I. Siddiqi, Phys. Rev. X 7, 031023 (2017).

[28] O. Viyuela, A. Rivas, S. Gasparinetti, A. Wallraff, S. Filipp, and M. A. Martin-Delgado, npj Quantum Inf. 4, 10 (2016).

[29] M. D. Schroer, M. H. Kolodrubetz, W. F. Kindel, M. Sandberg, J. Gao, M. R. Vissers, D. P. Pappas, A. Polkovnikov, and K. W. Lehnert, Phys. Rev. Lett. 113, 050402 (2014).
[30] P. Roushan, C. Neill, Y. Chen, M. Kolodrubetz, C. Quintana, N. Leung, M. Fang, R. Barends, B. Campbell, Z. Chen, B. Chiaro, A. Dunsworth, E. Jeffrey, J. Kelly, A. Megrant, J. Mutus, P. J. O’Malley, D. Sank, A. Vainsencher, J. Wenner, T. White, A. Polkovnikov, A. N. Cleland, and J. M. Martinis, Nature (London) 515, 241 (2014).

[31] V. Gritsev and A. Polkovnikov, Proc. Natl. Acad. Sci. USA 109, 6457 (2012).

[32] T. Albash and D. A. Lidar, Rev. Mod. Phys. 90, 015002 (2018).

[33] W. P. Su, J. R. Schrieffer, and A. J. Heeger, Phys. Rev. Lett. 42, 1698 (1979).

[34] J. Fields, H. W. Blöte, and J. C. Bonner, J. Appl. Phys. 50, 1807 (1979).

[35] R. Cleve, A. Ekert, C. Macchiavello, and M. Mosca, Proc. R. Soc. London A 454, 339 (1998).

[36] M. Dobšíček, G. Johansson, V. Shumeiko, and G. Wendin, Phys. Rev. A 76, 030306(R) (2007).

[37] A. Peruzzo, J. McClean, P. Shadbolt, M.-H. Yung, X.-Q. Zhou, P. J. Love, A. Aspuru-Guzik, and J. L. O'Brien, Nat. Commun. 5, 4213 (2014).

[38] See Supplemental Material at http://link.aps.org/supplemental/ 10.1103/PhysRevA.101.020302 for a detailed description of the algorithm proposed in this Rapid Communication. The Supplemental Material also includes an in-depth discussion of the implementation of the simulations. In particular, for both the single-particle and interacting models, the basis-gate and Trotter-Suzuki decompositions are presented. For the specific case of the single-particle model, the correspondence between the Berry phase and the winding number is explained, and the properties of the quantum processor used are provided. As for the interacting model, the presence of finite-size effects is illustrated.

[39] J. Zak, Phys. Rev. Lett. 62, 2747 (1989).

[40] https://github.com/Qiskit/ibmq-device-information/tree/ master/backends/melbourne/V1.

[41] H. Bethe, Z. Phys. 71, 205 (1931).

[42] H. Bethe, in Selected Works of Hans A. Bethe: With Commentary (World Scientific, Singapore, 1997), pp. 155-183.

[43] J. Lado, R. Ortiz, and J. Fernandez-Rossier, in Graphene Nanoribbons, edited by L. Brey, P. Seneor, and A. Tejeda (IOP Press, Oxford, UK, 2019), Chap. 4.

[44] P. W. Anderson, Phys. Rev. 115, 2 (1959).

[45] T. Moriya, Phys. Rev. 120, 91 (1960).

[46] V. V. Shende, S. S. Bullock, and I. L. Markov, IEEE Trans. Comput.-Aided Des. Integr. Circuits Syst. 25, 1000 (2006).

[47] H. F. Trotter, Proc. Am. Math. Soc. 10, 4 (1959).

[48] M. Suzuki, Phys. Lett. A 146, 319 (1990).

[49] M. Nielsen and I. L. Chuang, Quantum Computation and Quantum Information: 10th Anniversary Edition (Cambridge University Press, Cambridge, UK, 2010).

[50] S. McArdle, X. Yuan, and S. Benjamin, Phys. Rev. Lett. 122, 180501 (2019).

[51] M. V. Berry, J. Phys. A: Math. Theor. 42, 365303 (2009).

[52] B. Murta and J. Fernández-Rossier (unpublished).

[53] J. G. E. Farhi and S. Gutmann, arXiv:1411.4028. 